\title{
Generalized anxiety and sleep quality among health care professionals during the COVID-19 pandemic: a cross-sectional study from a tertiary healthcare institution in Eastern India
}

\author{
Bijaya Nanda Naik ${ }^{\oplus}$, Sanjay Pandey ${ }^{\oplus}$, Rajath Rao ${ }^{\circledR}$, Manisha Verma ${ }^{\oplus}$, Prashant Kumar Singh ${ }^{\circledR}$
}

Department of Community and Family Medicine, All India Institute of Medical Sciences, Patna, India

Received: November 23, 2021

Revised: December 6, 2021

Accepted: January 10, 2022

Corresponding author:

Rajath Rao

Department of Community and Family Medicine, All India Institute of Medical Sciences, 106, First Floor, Academic Block, Patna 801507, India E-mail: urrr16@gmail.com

\section{ABSTRACT}

Objectives: With the emergence of the coronavirus disease 2019 (COVID-19) pandemic, healthcare professionals (HCPs) have experienced high levels of stress and anxiety because of the high risk of infection for themselves and their families. This has led to acute sleep problems for HCP. This study was designed to assess the anxiety and sleep quality of HCPs during the COVID-19 pandemic.

Methods: This cross-sectional study analyzed 370 HCPs employed at All India Institute of Medical Sciences Patna over 3 months, using the standard Generalized Anxiety Disorder 7-item scale (GAD-7) for suspected GAD and the Pittsburgh Sleep Quality Index for sleep quality. Results were tabulated and multivariable binomial logistic regression analysis was done to determine the predictors of poor sleep. Significance was attributed to $p<0.05$.

Results: Of the 370 HCPs screened, 52 (14.1\%; 95\% confidence interval [CI], 10.8\%-18.1\%) were found to have GAD and 195 (52.7\%; 95\% CI, 47.5\%-57.9\%) were found to be poor sleepers. The presence of any addictive habit (adjusted odds ratio [AOR], 1.833; 95\% CI, 1.12-2.8), unprotected contact with COVID-19 cases (AOR, 1.902; 95\% CI, 1.1-3.3), and the presence of GAD (AOR, 5.57; 95\% CI, 2.5-12.4) were found to be predictors of poor sleep quality among HCPs.

Conclusion: A significant proportion of HCPs were found to have suspected GAD and were poor sleepers. This highlights the need for measures to confront this problem.

Keywords: Anxiety; Anxiety disorders; COVID-19; Sleep disorders; Sleep quality

\section{Introduction}

India is one of the countries most affected by the coronavirus disease 2019 (COVID-19) pandemic. On November 1, 2020, India reported 8,223,000 confirmed cases of COVID-19, rising 
to $10,600,000$ by the end of January 2021 , with a death toll reaching 153,000 (110 deaths per million population) $[1,2]$. Not only has COVID-19 caused substantial social and economic disruptions, but it also remains a serious challenge for the health care system and other public services. From day 1 of the COVID-19 pandemic, frontline workers have been working day and night to help mitigate the impact of the pandemic and control its spread. Frontline workers include healthcare personnel, personnel involved in maintaining law and order, and workers involved in the supply and distribution of essential goods [3].

Health care professionals (HCPs) need a special mention because of their direct exposure through involvement in the assessment, quarantine, isolation, and treatment of confirmed COVID-19 cases. They are at constant risk of contracting the virus from their patients and fear the possibility of transmitting infections to their family members and being unable to safeguard their families from this pandemic. This stress may lead to acute sleep problems, including poor sleep quality and short sleep duration. Because of the unpredictable nature of severe acute respiratory syndrome coronavirus-2 (SARS-CoV-2) transmission and the novel nature of COVID-19, mitigation, prevention and control, and management strategies are ever-evolving. The absence of clear-cut strategies during the initial stages of the COVID-19 pandemic, together with the high risk of exposure, has added to the stress levels of HCPs [4].

Infectious disease outbreaks, such as the current COVID-19 pandemic, are associated with major psychological distress and have significant impacts on mental illness [5-7]. HCPs often experience sleep problems, anxiety, depression, and stress when facing major public health threats $[8,9]$. Career anxiety, job insecurity, decreased job satisfaction, and increased organizational and professional turnover have been positively associated with the COVID-19 fears of HCPs [10]. Reports in the literature state that 31\% to $50 \%$ of physicians have poor sleep quality [11-13]. HCPs are under stress in general due to the nature of their job, dealing with sickness, suffering, and death. They often have irregular work schedules and frequent long shifts, leading to burnout, which may adversely impact their sleep [14-17]. A systematic review from Africa revealed that 9.5\% to $73.3 \%$ of HCPs suffered from anxiety disorders and $12.5 \%$ to $71.9 \%$ dealt with depression [18].

Few studies have concentrated on the sleep quality of HCPs during pandemic events, in particular the outbreak of COVID-19 in India [17,19]. This study was conducted during the first wave of the COVID-19 pandemic in India when resources were scarce. All India Institute of Medical
Sciences (AIIMS) Patna is a dedicated COVID-19 facility in the eastern state of Bihar, India, with a 500-bed capacity earmarked for COVID-19 patients during the pandemic. The HCPs, including all categories of healthcare workers, have made nonstop efforts to provide the best possible healthcare to the COVID-19 patients and to help control the pandemic in Bihar. In this context, the current study was designed to assess the anxiety levels and sleep quality of HCPs working at AIIMS Patna, India during the COVID-19 pandemic. In addition, we identified the factors associated with poor sleep quality among HCPs.

\section{Materials and Methods}

\section{Study Design and Duration}

This was a hospital-based, cross-sectional study that was carried out for a duration of 3 months (November 2020 to January 2021).

\section{Study Setting}

This study was conducted at AIIMS Patna, an Institute of National Importance under the Ministry of Health and Family Welfare, Government of India. AIIMS Patna is engaged in providing comprehensive healthcare to the people of Bihar. This institute was declared a dedicated COVID-19 hospital during the pandemic with a 500-bed capacity for serving the people of Bihar and neighboring states.

\section{Study Population}

The study's population included all HCPs involved in COVID-19 management, primarily doctors (medical faculty, senior residents, junior residents, interns) and nursing officers. HCPs who were on psychiatric medications at the time of the study (self-reported) were excluded.

\section{Sample Size and Sampling Technique}

With a 43.9\% prevalence of poor sleep quality among HCPs during the COVID-19 pandemic [20], the minimum sample size was calculated to be 218 at $15 \%$ relative precision and 95\% confidence interval (CI). The final sample size was calculated to be 242 after considering a $10 \%$ non-response rate. Nonetheless, we intended to include all eligible employees at our institution in the study.

The list of all healthcare employees of AIIMS Patna and their contact numbers were obtained from the administrative section. After all staff nurses and doctors involved in COVID-19 management in any capacity were identified and their contact details (WhatsApp number/ email) were collected, the study tool was shared and they 
were asked to participate voluntarily. A total of 370 HCPs participated in the study.

\section{Study Tool and Technique}

Information was collected using a pre-designed, standard questionnaire on Google Forms that was sent to the eligible participants via WhatsApp and email. Digital consent was incorporated into the Google Forms link, and participants could proceed only after giving consent.

The questionnaire had 3 sections and all questions were in English. Section A included the sociodemographic details section $B$ included questions to screen for anxiety using the Generalized Anxiety Disorder 7-item scale (GAD7) [21], and section $C$ included questions about subjective sleep quality using the Pittsburgh Sleep Quality Index (PSQI) [22]. The GAD-7 scale includes 7 questions and each question is scored $0-3$, with 0 indicating "not at all sure" and 3 indicating "nearly every day." The overall score for the GAD-7 scale ranges from 0 to 21. Scores of 5, 10, and 15 are used as the cut-off points for mild, moderate, and severe anxiety, respectively. Participants with a threshold score of $\geq 10$ are considered to have generalized anxiety disorder, and further evaluation is recommended. This cut-off was found to be $85 \%$ sensitive and $82 \%$ specific in screening for generalized anxiety disorder, with excellent internal consistency (Cronbach alpha, 0.92) and good test-retest reliability (interclass correlation coefficient, 0.83) [21].

The PSQI includes questions related to usual sleep habits during the past month divided into 7 categories: subjective sleep quality, sleep latency, sleep duration, habitual sleep efficiency, sleep disturbances, use of sleep medications, and daytime dysfunction. Overall sleep quality was rated as very good, fairly good, fairly bad, and very bad. Scores were given to each category $(0-3)$ and the global score ranged from 0 to 21. High scores indicate increased sleeping disturbance. Participants with scores $\geq 5$ are considered poor sleepers. The cut-off of $\geq 5$ had a diagnostic sensitivity of $89.6 \%$ and specificity of $86.5 \%$ for distinguishing poor sleepers and an overall reliability coefficient (Cronbach alpha) of 0.83 [22].

\section{Statistical Analysis}

The collected information was entered in MS Excel and the statistical analysis was done using IBM SPSS ver. 22.0 (IBM Corp., Armonk, NY, USA) Descriptive analyzes were conducted to describe the demographic characteristics and COVID-19-related data of HCPs who treated COVID-19 patients. Categorical variables such as sex, presence of generalized anxiety disorder, and poor sleeping were expressed as proportions and percentages. Continuous variables such as GAD-7 scores and PSQI scores were expressed as the median and interquartile range (IQR). The median difference in the GAD-7 scores among various groups was assessed using the Mann-Whitney U-test/ Kruskal-Wallis test. A multivariable binomial logistic regression analysis was done to determine the predictors of suspected GAD and poor sleep quality among the HCP. Statistical significance was attributed to $p<0.05$

\section{Ethical Considerations}

This study was approved by the Institute Ethics Committee, AIIMS, Patna (AIIMS/Pat/IEC/2020/697). The principles of ethics were adhered to throughout the study and thereafter.

\section{Results}

\section{General Characteristics of Study Participants}

The mean age of the HCPs was $28.8 \pm 5.2$ years. Among the $370 \mathrm{HCP}, 246$ (66.5\%) were male, 247 (66.8\%) lived offcampus in their own homes, 187 (50.5\%) were nursing staff, and 168 (45.4\%) had at least 1 addictive habit (of those, 25 [14.9\%] used tobacco). A total of 22 participants (5.9\%) had a history of psychiatric disorders but were not under any antipsychotic medication/care at the time of the study. We found that 77 (20.8\%) had been in contact with a COVID-19 patient without any protection (Table 1).

Among the 370 HCPs, 196 (53.0\%) had no symptoms related to COVID-19 for 1 month before the survey. Of the 174 HCPs (47.0\%) who had symptoms, 103 (59.2\%) had a cough, 100 (57.5\%) had fever, 91 (52.3\%) had body aches, 55 (31.6\%) had a loss of appetite, and 33 (19.0\%) had a loss of smell and/ or taste sensation. A total of 71 participants (19.2\%) reported testing positive for COVID-19 prior to or during the study period (Table 1).

\section{Generalized Anxiety Disorder among HCPs (GAD-7)}

The GAD-7 scores of the participants are provided in Table 1. The median (IQR) global GAD-7 score was 2.5 (0-6). HCPs with a history of psychiatric illness had a median (IQR) score of 5 (0-10.5). There were statistically significant differences in the median (IQR) scores when comparing HCPs with high-risk persons in their family to those without (3 [1-7] vs. $2[0-5], p<0.001)$ and when comparing those who tested positive for COVID-19 to those who tested negative (3 [1-8] vs. 2 [0-5], $p<0.02$ ) (Table 1 ).

Among all participants, 246 (66.5\%) had minimal/ no anxiety, 72 (19.5\%) had mild anxiety, 37 (10.0\%) had moderate anxiety, and 15 (4.1\%) had severe anxiety. We found that 12 interns (34.3\%), 30 junior residents (32.6\%), 7 senior residents (20.0\%), 8 faculty (38.1\%), and 52 nursing staff (27.8\%) had mild to moderate anxiety (Figure 1). Overall, 
Table 1. Sociodemographic details and distribution of the global GAD-7 and PSQI scores of HCPs $(n=370)$

\begin{tabular}{|c|c|c|c|}
\hline \multirow{2}{*}{ Variable } & \multirow{2}{*}{$n(\%)$} & GAD-7 scores & Global PSQI scores \\
\hline & & \multicolumn{2}{|c|}{ Median (IQR) } \\
\hline & & & \\
\hline \multicolumn{4}{|l|}{ Sex } \\
\hline Male & $246(66.5)$ & $2(0-5)$ & $5(3-7)$ \\
\hline Female & $124(33.5)$ & $3(0-6.5)$ & $5(3-7)$ \\
\hline \multicolumn{4}{|l|}{ Residence } \\
\hline Dormitory & $123(33.2)$ & $3(1-7)$ & $5(3-7)$ \\
\hline Own home & $247(66.8)$ & $2(0-6)$ & $5(3-7)$ \\
\hline \multicolumn{4}{|l|}{ Designation } \\
\hline Interns & $35(9.5)$ & $3(0-7)$ & $4(3-6)$ \\
\hline Junior resident & $92(24.9)$ & $3(1-7)$ & $5(3-7)$ \\
\hline Senior resident & $35(9.5)$ & $2(0-5)$ & $5(2-7)$ \\
\hline Medical faculty & $21(5.7)$ & $5(0.5-12.5)$ & $6(2.5-11)$ \\
\hline Nursing staff & $187(50.5)$ & $2(0-5)$ & $5(3-7)$ \\
\hline \multicolumn{4}{|l|}{ Addictive habits $^{\text {a) }}$} \\
\hline No & $202(54.6)$ & $2(0-5)$ & $4(3-6)$ \\
\hline Yes & $168(45.4)$ & $3(0-7)$ & $5(3-7.5)$ \\
\hline Tobacco $(n=168)^{a, b)}$ & $25(14.9)$ & $4(0.5-12)$ & $6(5-9)$ \\
\hline Beverages $(n=168)^{\mathrm{a})}$ & $152(90.5)$ & $3(0-7)$ & $5(3-8)$ \\
\hline Other habits $(n=168)$ & $13(7.7)$ & $2(0-6)$ & $4(2-6)$ \\
\hline \multicolumn{4}{|c|}{ History of psychiatric illness } \\
\hline No & $348(94.1)$ & $2(0-6)$ & $5(3-7)$ \\
\hline Yes & $22(5.9)$ & $5(0-10.5)$ & $5(4-8.5)$ \\
\hline \multicolumn{4}{|l|}{ High-risk family member ${ }^{\mathrm{a}, \mathrm{b}}$ ) } \\
\hline No & $204(55.1)$ & $2(0-5)$ & $4(3-6)$ \\
\hline Yes & $166(44.9)$ & $3(1-7)$ & $5(3-8)$ \\
\hline \multicolumn{4}{|c|}{ Unprotected contact with COVID-19 positive patient ${ }^{\mathrm{a}, \mathrm{b})}$} \\
\hline No & $227(61.4)$ & $2(0-5)$ & $4(2-6)$ \\
\hline Yes & $77(20.8)$ & $4(1-9)$ & $6(3.5-9)$ \\
\hline Don't know & $66(17.8)$ & $3(0-7)$ & $5(3-8)$ \\
\hline \multicolumn{4}{|c|}{ Tested positive for Covid- $19^{a, b)}$} \\
\hline No & $258(69.7)$ & $2(0-5)$ & $4(3-6)$ \\
\hline Yes & $71(19.2)$ & $3(1-8)$ & $5(3-9)$ \\
\hline Was not tested & $41(11.1)$ & $3(0-6.5)$ & $5(3.5-7)$ \\
\hline
\end{tabular}

GAD-7, Generalized Anxiety Disorder 7-item scale; PSQI, Pittsburgh Sleep Quality Index; HCP, healthcare professional; IQR, interquartile range; SD, standard deviation; COVID-19, coronavirus disease 2019.

${ }^{a}$ Statistically significant $(p<0.05)$ for median $(I Q R)$ global PSQI scores using the Mann-Whitney U-test for presence of any addictive habit, tobacco consumption, and the presence of a high-risk group in the family and using the Kruskal-Wallis test for unprotected contact with a COVID-19 positive patient and testing positive for COVID-19. ${ }^{\text {b) }}$ Statistically significant $(p<0.05)$ for median (IQR) global GAD-7 scores using the Mann-Whitney U-test for tobacco consumption and the presence of high-risk group in the family and using the Kruskal-Wallis test for unprotected contact with a COVID-19 positive patient and testing positive for COVID-19.

a total of 52 (14.1\%; 95\% CI, 10.8-18.1) out of 370 HCPs were screened and found to have suspected generalized anxiety disorder.

\section{Sleep Quality among HCPs (PSQI)}

The PSQI scores of participants are presented in Table 1. The median (IQR) global PSQI score was 5 (3-7). There was a statistically significant difference in the median scores when comparing HCPs who: (1) had high-risk persons in their family (median [IQR] PSQI score: 5 [3-8]) to those who did not (median [IQR] PSQI score: 4 (3-6); $p<0.001$ ); (2) those who had unprotected contact with COVID-19 cases (median [IQR] PSQI score: 6 [3.5-9]) to those who did not (median [IQR] PSQI score: 4 [2-6]; $p<0.001$ ); and (3) those who tested positive for COVID-19 (median [IQR] PSQI score: 5 [3-9]) to those who tested negative (median [IQR] PSQI score: 4 [3-6]; 
Minimal anxiety (0-4)

Moderate anxiety (10-14)
Mild anxiety (5-9)

Severe anxiety (16-21)

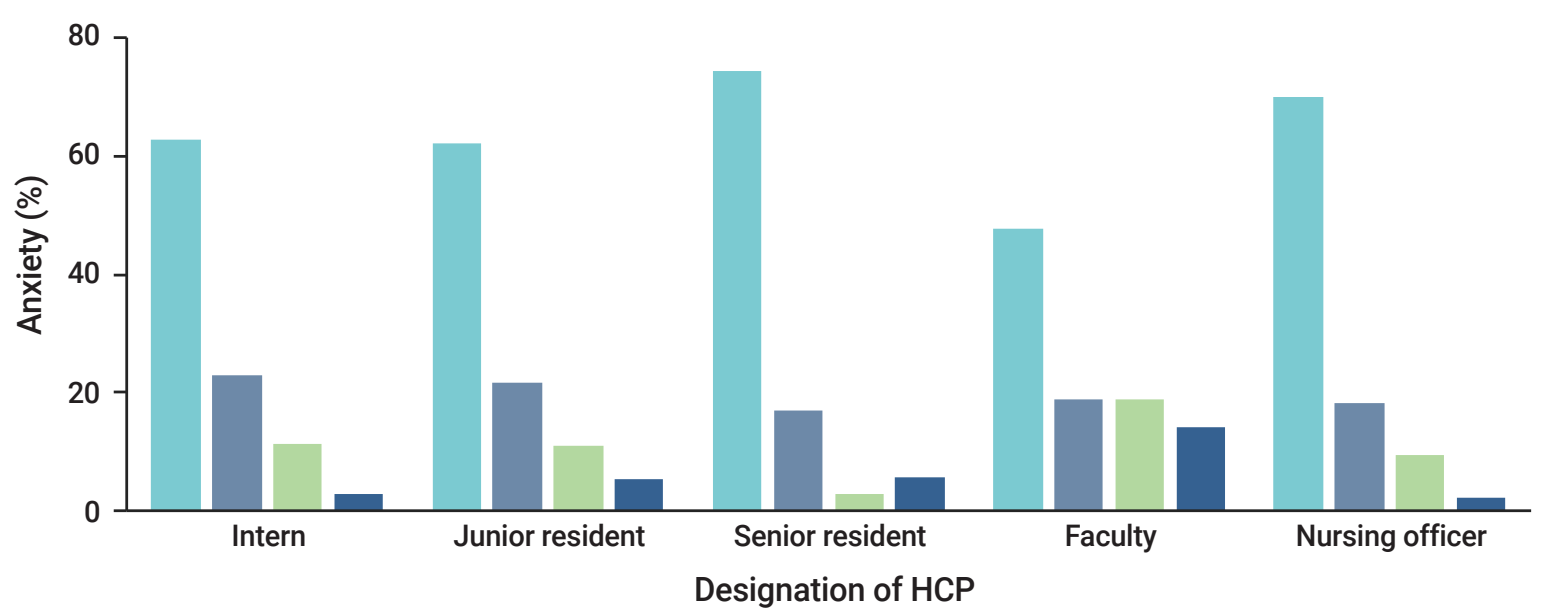

Figure 1. Distribution of the severity levels of generalized anxiety disorder among different healthcare professionals (HCPS).

$p=0.02)$ (Table 1).

The median (IQR) PSQI scores of HCPs (maximum score of 3) were as follows: sleep latency, 1 (1-2); sleep duration, 1 (0-2); sleep disturbances, 1 (0-1); daytime dysfunction, 1 (1-2); and 0 out of a maximum score of 3 for subjective sleep quality, habitual sleep efficiency, and use of sleep medicines.

Among the HCPs, 296 (80.0\%) had altered sleep latency, $263(71.1 \%)$ had altered sleep duration, 236 (63.8\%) had impaired habitual sleep efficiency, and 289 (78.1\%) had daytime dysfunction (Figure 2). Overall, more than half of the HCPs ( $n=195,52.7 \%$; $95 \%$ CI, 47.5\%-57.9\%) were found to be poor sleepers.

\section{Predictors of Suspected Generalized Anxiety Disorder}

Using univariate analysis, the presence of high-risk persons in the family was found to be a significant predictor of suspected GAD (unadjusted odds ratio [OR], 2.42; 95\% CI, 1.3-4.4). However, other variables including the presence of addictive habits, a history of psychiatric illness, unprotected contact with COVID-19 cases, and having tested positive for COVID-19 were also considered in multivariable logistic regression.

The presence of high-risk persons (elders, children, pregnant/lactating mothers, those with chronic diseases) in the family was found to be an independent predictor of suspected generalized anxiety disorder (adjusted OR [AOR], 2.22; $95 \%$ CI, 1.2-4.1), with a predictive accuracy of $85.9 \%$ (Table 2).

\section{Predictors of Poor Sleep Quality}

In the univariate analysis, the presence of addictive habits (crude OR, 1.982; 95\% CI, 1.3-3.007), unprotected contact with COVID-19 cases (crude OR, 2.182; 95\% CI, 1.2-3.7), and suspected generalized anxiety disorder (crude OR, 6.083; 95\% CI, 2.7-13.3) were found to be significant predictors of poor sleep quality. These variables, along with the presence of high-risk persons in the family, were considered in multivariable logistic regression.

The presence of an addictive habit (AOR, 1.833; 95\% CI, $1.1-2.8 ; p=0.006)$, history of unprotected contact with a COVID-19 case (AOR, 1.902; 95\% CI, 1.1-3.3; $p=0.02$ ), and suspected generalized anxiety disorder (AOR, 5.57; 95\% CI, 2.5-12.4; $p<0.001$ ) were found to be independent predictors of poor sleep among HCPs, with a predictive accuracy of $62.4 \%$ (Table 3).

\section{Discussion}

Sleep quality and anxiety levels are important concerns for HCPs since these issues can impact their performance and result in poor patient outcomes [23,24]. Sleep problems are, in turn, associated with psychological distress [13]. During the COVID-19 pandemic, HCPs have been overburdened with work and the risk of contracting SARS-CoV-2 remains high. An Indian Council of Medical Research serosurvey conducted during December 2020 and January 2021 reported that almost one-fourth (25\%) of healthcare workers showed evidence of SARS-COV-2 infection [25]. It is important to determine the proportion of HCPs with GAD and poor 


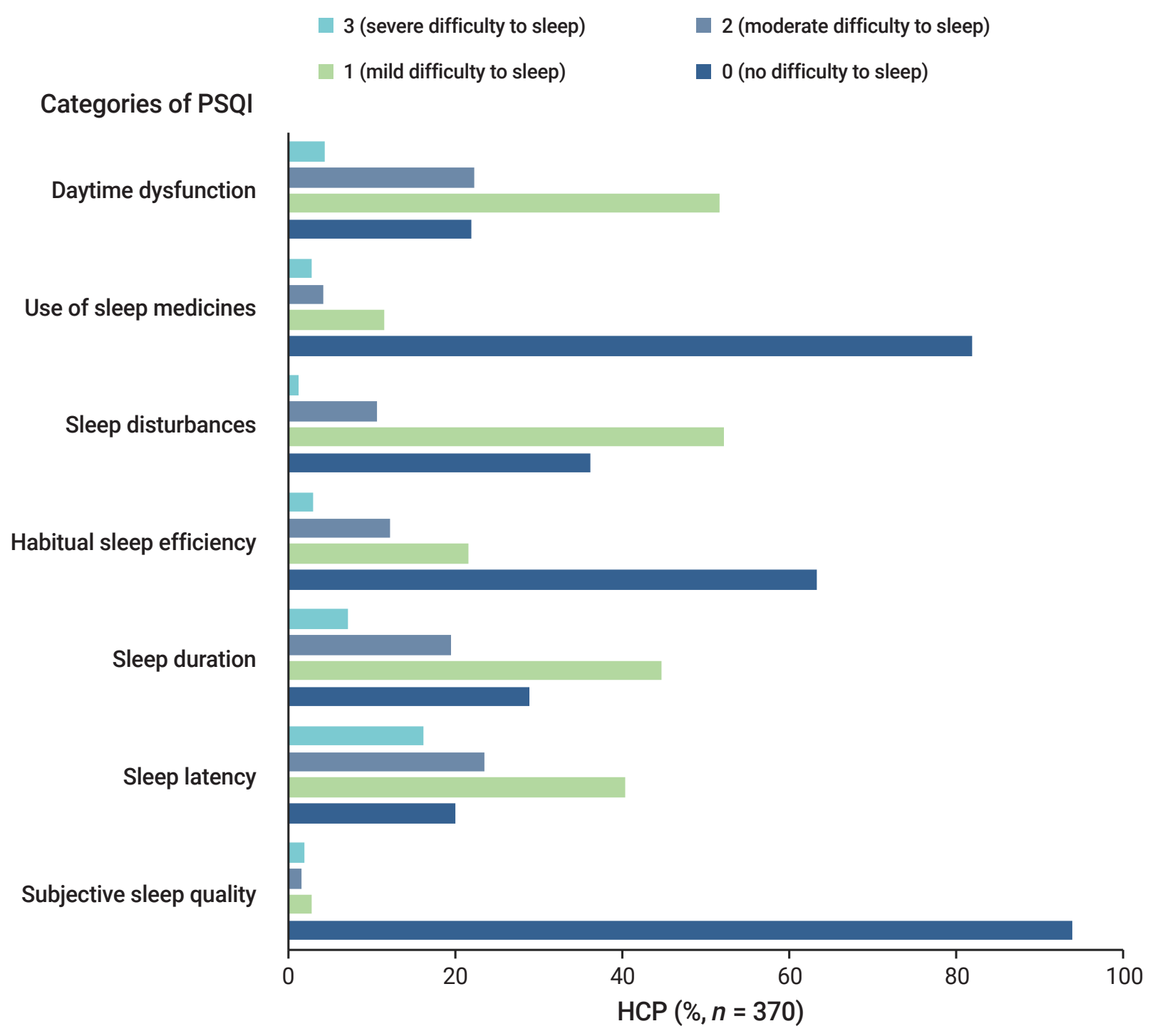

Figure 2. Distribution of varying grades of sleep difficulty among healthcare professionals (HCPs), across various categories of the Pittsburgh Sleep Quality Index (PSQI).

sleep in order to plan for future emergencies. Our crosssectional survey screened 370 HCPs working in a COVID-19 dedicated hospital and found the prevalence and predictors of suspected generalized anxiety disorder and poor sleep among HCP.

In our study, one-fifth of HCPs were exposed to COVID-19 without protection, whereas a study in China [26] reported only $2.7 \%$ exposure among HCPs. This may be due to the limited resources in our hospital, including personnel and beds, which led to higher exposure to SARS-COV-2 among HCPs.

The results of screening in our study showed that $14.1 \%$ of HCPs had suspected generalized anxiety disorder. Similar findings (e.g., a prevalence of anxiety among HCPs of 11.5\%) have been reported in other studies [27,28]. However, a study from Turkey reported that $39.4 \%$ of HCPs had anxiety [29]. In our study, $29.5 \%$ of HCPs had mild to moderate anxiety and $14.1 \%$ had severe anxiety, which is similar to a study from Saudi Arabia, which showed that more than $25 \%$ of HCPs had mild to moderate anxiety and nearly $10 \%$ had severe anxiety [30]. A study from southern Ethiopia reported a higher prevalence of mild to moderate anxiety among HCPs (35.6\%) [31]. The reported prevalence of anxiety among the Indian public is $28 \%$ [32]. Our study showed that the prevalence of mild to moderate anxiety in nursing staff was $27.8 \%$, while Meo et al. [30] reported almost double the anxiety (51.7\%) in the same group. Magnavita et al. [33], in a 1-year prospective study in Italy, 
Table 2. Predictors of suspected GAD among HCPs $(n=370)$

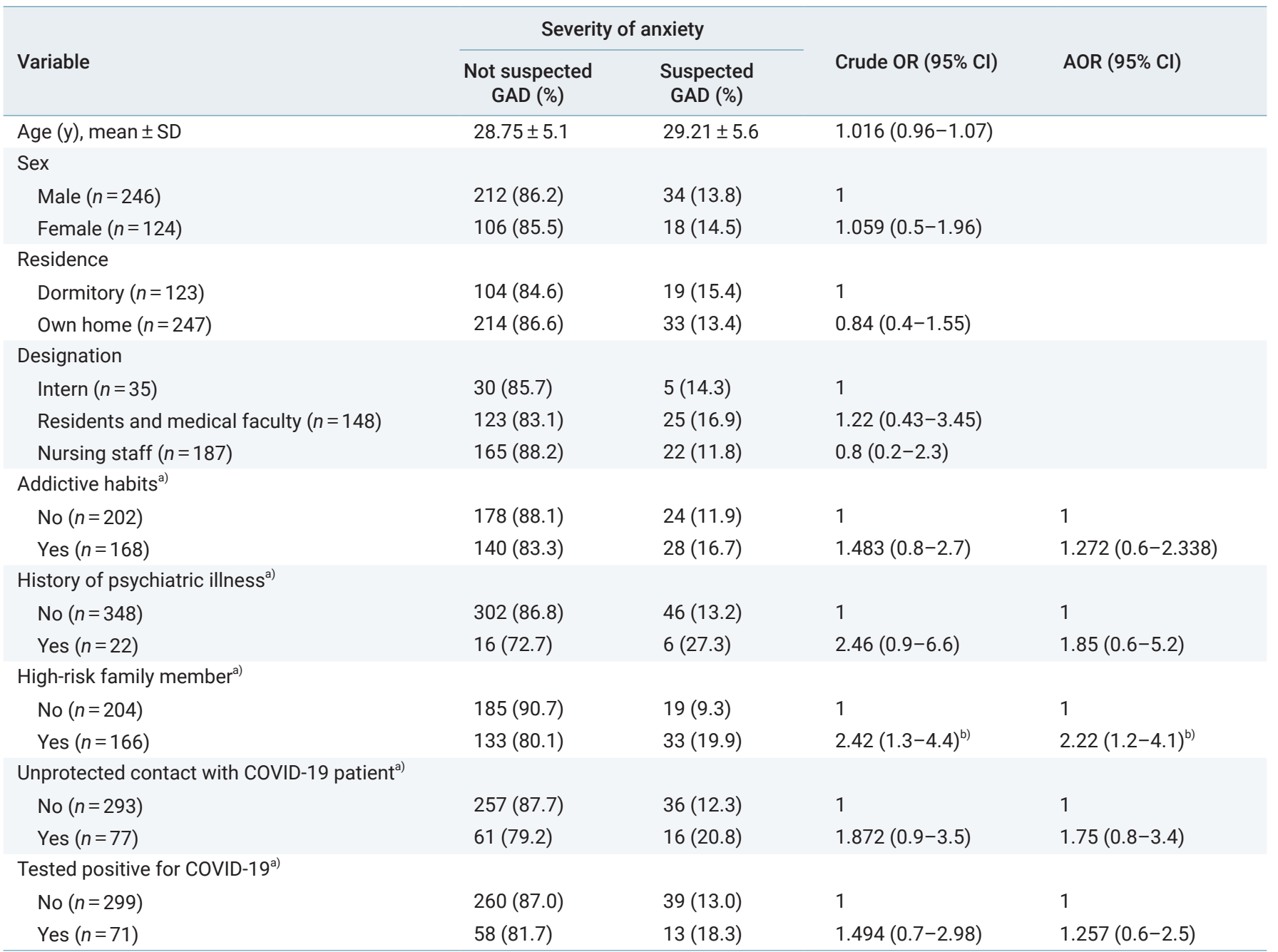

GAD, generalized anxiety disorder; HCP, healthcare professional; OR, odds ratio; Cl, confidence interval; AOR, adjusted OR; SD, standard deviation; COVID-19, coronavirus disease 2019.

a) Used for adjustment of the final model; Nagelkerke $\mathrm{R}^{2}=0.068(6.8 \%) .{ }^{\mathrm{b}} p<0.05$ indicates statistical significance.

reported that anxiety among $\mathrm{HCPs}$ was $25 \%$ and that even positive attitudes of doctors regarding the COVID-19 vaccine and procedural justice did not improve the mental health of HCPs.

The presence of high-risk family members was found to be an independent risk factor for anxiety among HCPs due to fear of unknowingly infecting their family members with SARS-CoV-2 and this was confirmed by another study on a large group of HCPs [34]. A study by Teshome et al. [31] in 2020 showed that factors such as contact with confirmed/ suspected COVID-19 cases, lack of access to COVID-19 media updates, the absence of confidence in one's ability to cope with the stresses of COVID-19, and other COVID-related worries increased the rate of anxiety disorder among HCPs. A systematic review from Africa showed that the availability of personal protective equipment and information regarding preventive measures reduced anxiety among HCPs, while substance use, history of chronic illness, low resilience, and low social support increased the odds of anxiety [18]. Another study from Iran predicted that less work experience, high levels of exposure to COVID-19 patients, having a history of mental illness, and underlying medical conditions increased the odds of higher anxiety among HCPs [35].

In our study, almost half of the HCPs (52.7\%) were found to be poor sleepers during this pandemic, which is in line with a study from China and a multicenter study from India, China, and Nepal $[26,36]$. The prevalence of sleep disorders during the COVID-19 pandemic was reported to range from $18.5 \%$ to $61 \%$ [37-39]. However, a study from 
Table 3. Predictors of poor quality of sleep among HCPs $(n=370)$

\begin{tabular}{|c|c|c|c|c|}
\hline \multirow[b]{2}{*}{ Variable } & \multicolumn{2}{|c|}{ Quality of sleep } & \multirow[b]{2}{*}{ Crude OR $(95 \% \mathrm{Cl})$} & \multirow[b]{2}{*}{ AOR $(95 \% \mathrm{Cl})$} \\
\hline & $\begin{array}{l}\text { Good sleepers } \\
\qquad(n=175, \%)\end{array}$ & $\begin{array}{l}\text { Poor sleepers } \\
\quad(n=195, \%)\end{array}$ & & \\
\hline Age $(y)($ mean $\pm S D)$ & $28.7 \pm 5.4$ & $28.9 \pm 4.9$ & $1.008(0.9-1.04)$ & \\
\hline \multicolumn{5}{|l|}{ Sex } \\
\hline Male $(n=246)$ & $116(47.2)$ & $130(52.8)$ & & \\
\hline Female $(n=124)$ & $59(47.6)$ & $65(52.4)$ & $0.983(0.6-1.6)$ & \\
\hline \multicolumn{5}{|l|}{ Residence } \\
\hline Dormitory $(n=123)$ & $57(46.3)$ & $66(53.7)$ & $1.059(0.7-1.6)$ & \\
\hline Own home $(n=247)$ & $118(47.8)$ & $129(52.2)$ & & \\
\hline \multicolumn{5}{|l|}{ Designationa) $^{\text {a) }}$} \\
\hline Intern $(n=35)$ & $20(57.1)$ & $15(42.9)$ & & 1 \\
\hline Residents and medical faculty $(n=148)$ & $63(42.6)$ & $85(57.4)$ & $1.799(0.8-3.8)$ & $1.527(0.7-3.4)$ \\
\hline Nursing staff $(n=187)$ & $92(49.2)$ & $95(50.8)$ & $1.377(0.6-2.8)$ & $1.67(0.8-3.6)$ \\
\hline \multicolumn{5}{|l|}{ Addictive habits ${ }^{\text {a) }}$} \\
\hline No $(n=202)$ & $111(55.0)$ & $91(45.0)$ & & 1 \\
\hline Yes $(n=168)$ & $64(38.1)$ & $104(61.9)$ & $1.982(1.3-3.007)^{\mathrm{b})}$ & $1.833(1.1-2.8)(p=0.006)^{b)}$ \\
\hline \multicolumn{5}{|l|}{ History of psychiatric illness } \\
\hline No $(n=348)$ & $166(47.7)$ & $182(52.3)$ & 1 & \\
\hline Yes $(n=22)$ & $9(40.9)$ & $13(59.1)$ & $1.317(0.5-3.2)$ & \\
\hline \multicolumn{5}{|l|}{ High-risk family member ${ }^{a)}$} \\
\hline No $(n=204)$ & $104(51.0)$ & $100(49.0)$ & 1 & 1 \\
\hline Yes $(n=166)$ & $71(42.8)$ & $95(57.2)$ & $1.392(0.9-2.1)$ & $1.159(0.75-1.8)$ \\
\hline \multicolumn{5}{|l|}{ Unprotected contact with COVID-19 patient ${ }^{\text {a) }}$} \\
\hline No $(n=293)$ & $257(87.7)$ & $36(12.3)$ & 1 & 1 \\
\hline Yes $(n=77)$ & $25(32.5)$ & $52(67.5)$ & $2.182(1.2-3.7)^{b}$ & $1.902(1.1-3.3)(p=0.02)^{\mathrm{b})}$ \\
\hline \multicolumn{5}{|l|}{ Tested positive for COVID-19 } \\
\hline No $(n=299)$ & $145(48.5)$ & $154(51.5)$ & 1 & \\
\hline Yes $(n=71)$ & $30(42.3)$ & $41(57.7)$ & $1.287(0.7-2.1)$ & \\
\hline \multicolumn{5}{|l|}{ Suspected GAD } \\
\hline No $(n=52)$ & $167(52.5)$ & $151(47.5)$ & 1 & \\
\hline Yes $(n=318)$ & $8(15.4)$ & $44(84.6)$ & $6.083(2.7-13.3)^{\mathrm{b})}$ & $5.57(2.5-12.4)(p<0.001)^{\mathrm{b})}$ \\
\hline
\end{tabular}

HCP, healthcare professional; OR, odds ratio; Cl, confidence interval; AOR, adjusted OR; SD, standard deviation; COVID-19, coronavirus disease 2019; GAD, generalized anxiety disorder.

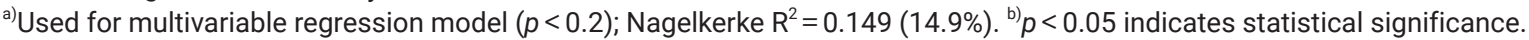

Turkey reported that up to $92 \%$ of surveyed HCPs were poor sleepers [40]. One in every 2 HCPs was reported to have poor sleep quality in a systematic review [11]. Nearly threequarters of frontline workers in Bahrain were reported to be poor sleepers [16].

The mean global PSQI score in our study was found to be $5.18 \pm 3.16$, which was lower than that found in a study in Riyadh, Saudi Arabia $(8.9 \pm 3.8)$ [30]. In our study, HCPs scored a median 1 out of 3 for each category of the PSQI (sleep latency, sleep duration, sleep disturbances, and daytime dysfunction), while a study by Jahrami et al. [16] showed mean PSQI scores of 1.6, 1, 1.3, and 1.1 out of 3 in these same categories, respectively.

Our study found that the presence of any addictive habits, unprotected contact with COVID-19 patients, and a positive screen for suspected generalized anxiety disorder were significant independent predictors of poor sleep and increased the odds of poor sleep quality among HCPs. A study from Turkey showed that the female sex, being a physician, and depression predicted poor sleep quality [29]. Another study from Taiwan reported that symptoms of anxiety, fear of COVID-19, and stress predicted poor sleep quality and insomnia [41]. In our study, the unadjusted odds of poor sleep for the nursing staff was 1.377, while a study by Zhou et al. [37] showed 3 times higher odds of poor sleep among nursing staff. Zhou et al. [37] also showed that older age and working in the emergency department were predictors of poor sleep quality among HCPs. In our study, resident 
doctors and faculty showed higher odds of poor sleep than others (interns and nursing staff). Factors influencing this could be working in an unfamiliar and chaotic work environment, a heavy clinical workload, burnout, and constant fear of infection for themselves and their family members. These biopsychosocial factors affect sleep quality [42]. It has been reported that sleep is the moderating factor in the relationship between occupational stress and anxiety [43].

Our study showed that HCPs with suspected generalized anxiety disorder had 5.7 times higher odds of poor sleep, which is in line with a study from Serbia done among HCPs treating COVID-19 patients [44].

All of these findings confirm the negative impact of the COVID-19 pandemic on the psychological well-being of HCPs in terms of severe GAD and poor sleep. The findings from our study will add to the limited but growing pool of literature on the mental health of HCPs during the COVID-19 pandemic.

\section{Conclusion}

A significant proportion of HCPs had suspected generalized anxiety disorder and were poor sleepers. The presence of a high-risk family member was the only significant independent predictor of suspected generalized anxiety disorder and the presence of addictive habits, unprotected contact with COVID-19 cases, and the presence of suspected generalized anxiety disorder were independent predictors of poor sleep quality among HCPs. This study provides further evidence concerning the negative impact of the COVID-19 pandemic on the mental health of HCPs. HCPs should be given optimum days off and the administration should provide the human resources necessary at times such as this pandemic to optimize mental well-being. HCPs also need ongoing institutional support regarding their roles and responsibilities, the risks involved, and resources available for self-protection. Emphasis should also be placed on the early detection of sleep problems among HCPs to help improve their ability to tackle future pandemics.

\section{Strength and Limitations}

To plan for the future, it is important to understand the anxiety, apprehension, workload, and sleep quality of HCPs, especially during an unprecedented situation like the COVID-19 pandemic, in order to provide an optimal level of healthcare without putting strain on the healthcare delivery workforce. This study is one of only a few to highlight the anxiety levels and sleep quality among HCPs in eastern India.
Our study was not without limitations. This was a study conducted at a single center with limited personnel, which is a situation similar to other parts of the country, considering the constraints on personnel in the healthcare sector. A causal relationship could not be established due to the cross-sectional design of the study. The self-reporting tool used for the collection of information may have led to some reporting bias, although the data collection was anonymous to ensure the privacy and confidentiality of information and to minimize reporting bias. This study did not differentiate pandemic-related anxiety and sleep problems from other causes of anxiety and sleep problems. The anxiety and sleep disorder results were probable and diagnostic using the screening questionnaires. However, the results clearly indicated that anxiety and sleep problems do exist among HCPs in the situation of the COVID-19 pandemic, and this problem requires attention and intervention.

\section{Notes}

\section{Ethics Approval}

This study was approved by the Institute Ethics Committee, AIIMS Patna (AIIMS/Pat/IEC/2020/697). We adhered to the principles of ethics throughout the study and thereafter and this study was conducted in accordance with the principles of the Declaration of Helsinki. An online survey was used and only those who gave consent to participate in the study were included.

\section{Conflicts of Interest}

The authors have no conflicts of interest to declare.

\section{Funding}

None.

\section{Availability of Data}

The datasets are not publicly available but are available from the corresponding author upon reasonable request.

\section{Authors' Contributions}

Conceptualization: BNN, SP; Data curation: RR, MV, PKS; Data analysis: RR; Methodology: BNN, RR; Supervision: BNN, SP; Writing-original draft: $\mathrm{RR}, \mathrm{PKS}$; Writing-review $\&$ editing: all authors.

\section{Acknowledgements}

We acknowledge the Department of Community and Family Medicine, AIIMS Patna for allowing this study and Junior residents from the department for helping in data collection process

\section{References}

1. World Health Organization (WHO) India. Novel coronavirus disease (COVID-19): situation update report-52 [Internet]. New Delhi: WHO India; 2021 [cited 2021 Dec 3]. Available from: https://cdn.who. int/media/docs/default-source/wrindia/situation-report/indiasituation-report-52.pdf?sfvrsn = cd0a4b84_4.

2. World Health Organization (WHO) India. Novel coronavirus disease 
(COVID-19): situation update report-40 [Internet]. New Delhi: WHO India; 2020 [cited 2021 Dec 3]. Available from: https://cdn.who. int/media/docs/default-source/wrindia/situation-report/indiasituation-report-40.pdf?sfvrsn $=477 b 71 b 5 \_88$ Status $=$ Master.

3. Centers for Disease Control and Prevention (CDC). Vaccines and immunizations: ACIP categories of essential workers [Internet]. Atlanta: CDC; 2021 [cited 2021 Nov 1]. Available from: https://www. cdc.gov/vaccines/covid-19/categories-essential-workers.html.

4. Grover S, Dua D, Sahoo S, et al. Why all COVID-19 hospitals should have mental health professionals: the importance of mental health in a worldwide crisis! Asian J Psychiatr 2020;51:102147.

5. Tucci V, Moukaddam N, Meadows J, et al. The forgotten plague: psychiatric manifestations of Ebola, Zika, and emerging infectious diseases. J Glob Infect Dis 2017;9:151-6.

6. Stewart-Ibarra AM, Hargrave A, Diaz A, et al. Psychological distress and Zika, dengue and chikungunya symptoms following the 2016 earthquake in Bahia de Caraquez, Ecuador. Int J Environ Res Public Health 2017;14:E1516.

7. Wang C, Pan R, Wan X, et al. Immediate psychological responses and associated factors during the initial stage of the 2019 coronavirus disease (COVID-19) epidemic among the general population in China. Int J Environ Res Public Health 2020;17:1729.

8. Cates DS, Gomes PG, Krasilovsky AM. Behavioral health support for patients, families, and healthcare workers. In: Hewlett A, Murthy ARK, editors. Bioemergency planning: a guide for healthcare facilities. Cham: Springer International Publishing; 2018. p. 195-214.

9. Levin J. Mental health care for survivors and healthcare workers in the aftermath of an outbreak. In: Huremovic D, editor. Psychiatry of pandemics: a mental health response to infection outbreak. Cham: Springer Nature Switzerland; 2019. p. 127-41.

10. Rajabimajd N, Alimoradi Z, Griffiths MD. Impact of COVID-19-related fear and anxiety on job attributes: a systematic review. Asian J Soc Health Behav 2021;4:51-5.

11. Qiu D, Yu Y, Li RQ et al. Prevalence of sleep disturbances in Chinese healthcare professionals: a systematic review and meta-analysis. Sleep Med 2020;67:258-66.

12. AlSaif HI. Prevalence of and risk factors for poor sleep quality among residents in training in KSA. J Taibah Univ Med Sci 2018;14:52-9.

13. Alimoradi Z, Brostrom A, Tsang HWH, et al. Sleep problems during COVID-19 pandemic and its' association to psychological distress: a systematic review and meta-analysis. EClinicalMedicine 2021; 36:100916.

14. Kumar DD. The relation of stressful life events and a happy life. Int J Soc Sci Interdiscip Res 2013;2:213-9.

15. Mohanty A, Kabi A, Mohanty AP. Health problems in healthcare workers: a review. J Family Med Prim Care 2019;8:2568-72.

16. Jahrami H, BaHammam AS, AlGahtani $\mathrm{H}$, et al. The examination of sleep quality for frontline healthcare workers during the outbreak of COVID-19. Sleep Breath 2021;25:503-11.

17. Patel BR, Khanpara BG, Mehta PI, et al. Evaluation of perceived social stigma and burnout, among health-care workers working in COVID-19 designated hospital of India: a cross-sectional study. Asian J Soc Health Behav 2021;4:156-62.

18. Olashore AA, Akanni OO, Fela-Thomas AL, et al. The psychological impact of COVID-19 on health-care workers in African countries: a systematic review. Asian J Soc Health Behav 2021;4:85-97.

19. Sharma R, Bansal P, Chhabra M, et al. Severe acute respiratory syndrome coronavirus-2-associated perceived stress and anxiety among indian medical students: a cross-sectional study. Asian J Soc Health Behav 2021;4:98-104.

20. Alnofaiey YH, Alshehri HA, Alosaimi MM, et al. Sleep disturbances among physicians during COVID-19 pandemic. BMC Res Notes 2020; 13:493.

21. Spitzer RL, Kroenke K, Williams JB, et al. A brief measure for assessing generalized anxiety disorder: the GAD-7. Arch Intern Med 2006;166: 1092-7.

22. Buysse DJ, Reynolds CF 3rd, Monk TH, et al. The Pittsburgh Sleep Quality Index: a new instrument for psychiatric practice and research. Psychiatry Res 1989;28:193-213.

23. Khatony A, Zakiei A, Khazaie H, et al. International nursing: a study of sleep quality among nurses and its correlation with cognitive factors. Nurs Adm Q 2020;44:E1-10.

24. LeBlanc VR. The effects of acute stress on performance: implications for health professions education. Acad Med 2009;84(10 Suppl):S25-33.

25. Murhekar MV, Bhatnagar T, Thangaraj JWV, et al. SARS-CoV-2 seroprevalence among the general population and healthcare workers in India, December 2020-January 2021. Int J Infect Dis 2021; 108:145-55.

26. Yang G, Li C, Zhu X, et al. Prevalence of and risk factors associated with sleep disturbances among HPCD exposed to COVID-19 in China. Sleep Med 2021;80:16-22.

27. Lai J, Ma S, Wang Y, et al. Factors associated with mental health outcomes among health care workers exposed to coronavirus disease 2019. JAMA Netw Open 2020;3:e203976.

28. Tan BYQ, Chew NWS, Lee GKH, et al. Psychological impact of the COVID-19 pandemic on health care workers in Singapore. Ann Intern Med 2020;173:317-20.

29. Arac S, Donmezdil S. Investigation of mental health among hospital workers in the COVID-19 pandemic: a cross-sectional study. Sao Paulo Med J 2020;138:433-40.

30. Meo SA, Alkhalifah JM, Alshammari NF, et al. Comparison of generalized anxiety and sleep disturbance among frontline and second-line healthcare workers during the COVID-19 pandemic. Int J Environ Res Public Health 2021;18:5727.

31. Teshome A, Glagn M, Shegaze M, et al. Generalized anxiety disorder and its associated factors among health care workers fighting COVID-19 in Southern Ethiopia. Psychol Res Behav Manag 2020;13:907-17.

32. Lakhan R, Agrawal A, Sharma M. Prevalence of depression, anxiety, and stress during COVID-19 pandemic. J Neurosci Rural Pract 2020;11:519-25.

33. Magnavita N, Soave PM, Antonelli M. A one-year prospective study 
of work-related mental health in the intensivists of a COVID-19 hub hospital. Int J Environ Res Public Health 2021;18:9888.

34. Barzilay R, Moore TM, Greenberg DM, et al. Resilience, COVID-19related stress, anxiety and depression during the pandemic in a large population enriched for healthcare providers. Transl Psychiatry 2020;10:291.

35. Hasannia E, Mohammadzadeh F, Tavakolizadeh M, et al. Assessment of the anxiety level and trust in information resources among iranian health-care workers during the pandemic of coronavirus disease 2019. Asian J Soc Health Behav 2021;4:163-8.

36. Khan HR, Ashraf F, Ullah I, et al. Cross-cultural prevalence of sleep quality and psychological distress in healthcare workers during COVID-19 pandemic. Brain Behav 2021;11:e2383.

37. Zhou Y, Yang Y, Shi T, et al. Prevalence and demographic correlates of poor sleep quality among frontline health professionals in Liaoning Province, China during the COVID-19 outbreak. Front Psychiatry 2020;11:520.

38. Que J, Shi L, Deng J, et al. Psychological impact of the COVID-19 pandemic on healthcare workers: a cross-sectional study in China. Gen Psychiatr 2020;33:e100259.
39. Ferini-Strambi L, Zucconi M, Casoni F, et al. COVID-19 and sleep in medical staff: reflections, clinical evidences, and perspectives. Curr Treat Options Neurol 2020;22:29.

40. Tasdemir Yigitoglu G, Yilmaz A, et al. The effect of Covid-19 on sleep quality, anxiety and depression on healthcare staff at a tertiary hospital in Turkey. Arch Psychiatr Nurs 2021;35:504-10.

41. Lu MY, Ahorsu DK, Kukreti S, et al. The prevalence of post-traumatic stress disorder symptoms, sleep problems, and psychological distress among COVID-19 frontline healthcare workers in Taiwan. Front Psychiatry 2021;12:705657.

42. Xiang YT, Jin Y, Wang Y, et al. Tribute to health workers in China: a group of respectable population during the outbreak of the COVID-19. Int J Biol Sci 2020;16:1739-40.

43. Magnavita N, Tripepi G, Di Prinzio RR. Symptoms in health care workers during the COVID-19 epidemic. a cross-sectional survey. Int J Environ Res Public Health 2020;17:5218.

44. Stojanov J, Malobabic M, Stanojevic G, et al. Quality of sleep and health-related quality of life among health care professionals treating patients with coronavirus disease-19. Int J Soc Psychiatry 2021;67:175-81. 